\title{
Relativistic Opacities for Astrophysical Applications
}

\author{
C.J. Fontes, C.L. Fryer, A.L. Hungerford, P. Hakel, J. Colgan, D.P. \\ Kilcrease, M.E. Sherrill \\ Los Alamos National Laboratory, Los Alamos, NM 87545, USA
}

\begin{abstract}
We report on the use of the Los Alamos suite of relativistic atomic physics codes to generate radiative opacities for the modeling of astrophysically relevant plasmas under local thermodynamic equilibrium (LTE) conditions. The atomic structure calculations are carried out in fine-structure detail, including full configuration interaction. Three example applications are considered: iron opacities at conditions relevant to the base of the solar convection zone, nickel opacities for the modeling of stellar envelopes, and samarium opacities for the modeling of light curves produced by neutron star mergers. In the first two examples, comparisons are made between opacities that are generated with the fully and semi-relativistic capabilities in the Los Alamos suite of codes. As expected for these highly charged, iron-peak ions, the two methods produce reasonably similar results, providing confidence that the numerical methods have been correctly implemented. However, discrepancies greater than $10 \%$ are observed for nickel and investigated in detail. In the final application, the relativistic capability is used in a preliminary investigation of the complicated absorption spectrum associated with cold lanthanide elements.
\end{abstract}

Keywords:

relativistic atomic data, LTE opacities, neutron star mergers

\section{Introduction}

As described in a recent review article [1], the Los Alamos SuitE of Relativistic (LASER) atomic physics codes has undergone significant development over the past twenty years. These codes can generate large-scale atomic physics models that are self-consistent and result in complex spectral features. As illustrated in that earlier work, the suite can been used to carry 
out detailed collisional-radiative (CR) modeling of plasmas under non-local thermodynamic equilibrium (non-LTE) conditions. In the present work, the relativistic suite is used to generate radiative opacities for LTE plasmas of astrophysical interest. A consideration of (simpler) LTE plasmas avoids the need to solve the system of coupled CR equations and therefore permits a more detailed, fine-structure treatment of the atomic models.

The methods used in the fundamental LASER atomic physics codes called RATS, ACE, and GIPPER [1] are largely based on the Penn State University codes developed by Sampson and coworkers [2]. The RATS atomic structure code is used to generate fully relativistic wavefunctions and energies within the Dirac-Fock-Slater approximation. These data are used to compute radiative rates, as well as electron-impact excitation (EIE) cross sections or collision strengths in the high-energy, plane-wave-Born approximation. The ACE code reads in data produced with RATS and is used to generate more accurate EIE data within the relativistic distorted-wave approximation. The GIPPER code also reads in data produced with RATS and is used to generate ionization cross sections and rates for the processes of electron-impact ionization, photoionization, and autoionization. The CR and kinetics modeling code called ATOMIC $[1,3,4,5]$ is then used to process the fundamental atomic data and generate spectra such as opacities and emissivities. Since the present work deals only with LTE opacity applications, EIE data are not required and so the ACE code was not used to generate any of the models. Similarly, of the three types of ionization processes mentioned above, only photoionization data are required for LTE opacities, which were calculated with the GIPPER code within the relativistic distorted-wave approximation.

In the following sections, we report on iron, nickel and samarium opacity calculations carried out with the LASER codes at conditions relevant for astrophysical plasmas of current interest. These models were generated in fine-structure detail with full configuration interaction. The iron and nickel calculations are compared with corresponding semi-relativistic results in a self-consistent manner in order to investigate the importance of relativistic and other effects. The semi-relativistic calculations were performed with the CATS code [1, 11], which is based on Cowan's atomic structure codes [12]. The samarium calculations are used in a preliminary assessment of the importance of lanthanide opacities to the light curves generated by neutron star mergers. In order to generate LTE level populations, we used the CHEMEOS package [4] for the iron and nickel cases, while simple Saha-Boltzmann statistics was used for the low-density samarium case. 


\section{Iron}

The iron-peak elements, typically defined as ranging from vanadium to nickel in the periodic table, are of long-established importance in the spectral diagnostics of astrophysical objects due to their relatively large and abundance spectral signatures. Iron itself has been the subject of extensive experimental campaigns designed to better understand certain spectral details of astrophysical relevance. Two such recent measurements were published in Nature, reporting significant discrepancies with theoretical calculations. The first article [6] concerned the ratio of two $3 d-2 p$ x-ray emission lines, referred to as $3 C$ and $3 D$, in Ne-like iron. The $3 \mathrm{C} / 3 \mathrm{D}$ line intensity ratio is an important diagnostic in interpreting a variety of astrophysical spectra, such as x-ray observations of stellar coronae [7].

In Ref. [6], a difference of 3.6 standard deviations was reported between the measured $3 \mathrm{C} / 3 \mathrm{D}$ oscillator strength ratio and state-of-the-art theoretical calculations, calling into question the validity of fundamental atomic structure calculations. This discrepancy has been largely resolved by using time-dependent rate equations to reinterpret the experimental results as a measured line intensity ratio rather than the more fundamental oscillator strength ratio $[8,9]$. The second, very recent article concerned the frequencydependent LTE opacity of iron under conditions associated with the base of the solar convection zone [10]. Large (30-400\%) differences were reported between experiment and theory for the monochromatic opacity of an iron plasma with a charge state distribution centered about the Ne-like stage. The source of this discrepancy is an outstanding mystery and remains an active area of research. In this context, we have performed fully relativistic (FR) calculations to compare against corresponding semi-relativistic (SR) in fine-structure mode with full configuration interaction. For such a highly charged iron plasma, good agreement between the Los Alamos FR and SR methods is expected, but in light of the present controversy, it is important to test such assumptions.

In this case we present a basic, proof-of-principle comparison, while a more complicated comparison is presented (for nickel) in the next section. The atomic physics model for this comparison was intentionally chosen to include a maximum principal quantum number of only $n_{\max }=4$, in contrast to a value of $n_{\max }=10$ that is typically used in our more standard, largescale efforts to generate LTE OPLIB tables $[13,14]$. The choice of $n_{\max }=4$ includes the most important contributions from configuration interaction for 
the strong bound-bound L-shell $(n=2-3)$ absorption lines that occur at these conditions, while keeping the calculation in a tractable form. The model contains eight ion stages centered about the Ne-like stage, ranging from C-like through Al-like. Single- and double-electron promotions were considered from the ground configuration of each ion stage with an occupied $n=2$ valence shell. For those ion stages with an occupied $n=3$ valence shell, up to three-electron promotions were considered. For all ion stages, a maximum of two electrons were allowed to exist in the $n=4$ shell, resulting in a total of approximately 220000 fine-structure levels. This is the same model that was considered in our earlier work [1], but the emphasis in that earlier work was to study the effect of varying amounts of configuration interaction. While the model is relatively small with respect to obtaining a converged spectrum, the use of full configuration interaction provides a sensitive, proofof-principle comparison of the FR and SR opacity-generating capabilities in the Los Alamos suite.

In Fig. 1, we present a comparison of FR and SR LTE monochromatic opacities, $\kappa_{\nu}$, of iron at a temperature of $T=193 \mathrm{eV}$ and an electron density of $N_{e}=10^{23}$ electrons $/ \mathrm{cm}^{3}$, similar to the conditions considered in Ref. [10]. As expected, the overall agreement between the two results is good, but the relatively small value of $n_{\max }=4$ causes significant differences in certain features. There are some large (15\%) differences for certain M-shell $(3 d-4 f$, $3 p-4 d$ ) features occurring around $200 \mathrm{eV}$. Large differences (35\%) also occur in the L-shell $(2 p-4 d)$ features above $1000 \mathrm{eV}$. The strongest L-shell $(2 s-3 p$, $2 p-3 d$ ) features below $1000 \mathrm{eV}$ agree very well because this $n_{\max }=4$ model contains most of the configuration interaction that is necessary to accurately describe the energies and wavefunctions associated with the $2-3$ transitions. The $2-4$ and $3-4$ transitions involve $n=4$ levels that are not completely converged in this model due to missing configuration interaction. The corresponding features should agree better between the two types of calculations as $n_{\max }$ is increased, and a more detailed study is reserved for future work.

The average charge state, $\bar{Z}$, and Rosseland mean opacity, $\kappa_{\mathrm{R}}$, for each calculation are also displayed in the legend of Fig. 1 . The $\bar{Z}$ values are identical to the precision shown. Highly averaged quantities, such as $\bar{Z}$, typically agree very well when comparing FR and SR models that were constructed from the same list of configurations, using the same level of resolution to generate the atomic data, such as the configuration-average or fine-structure method. The Rosseland mean opacity, $\kappa_{\mathrm{R}}$, is the relevant quantity of interest 


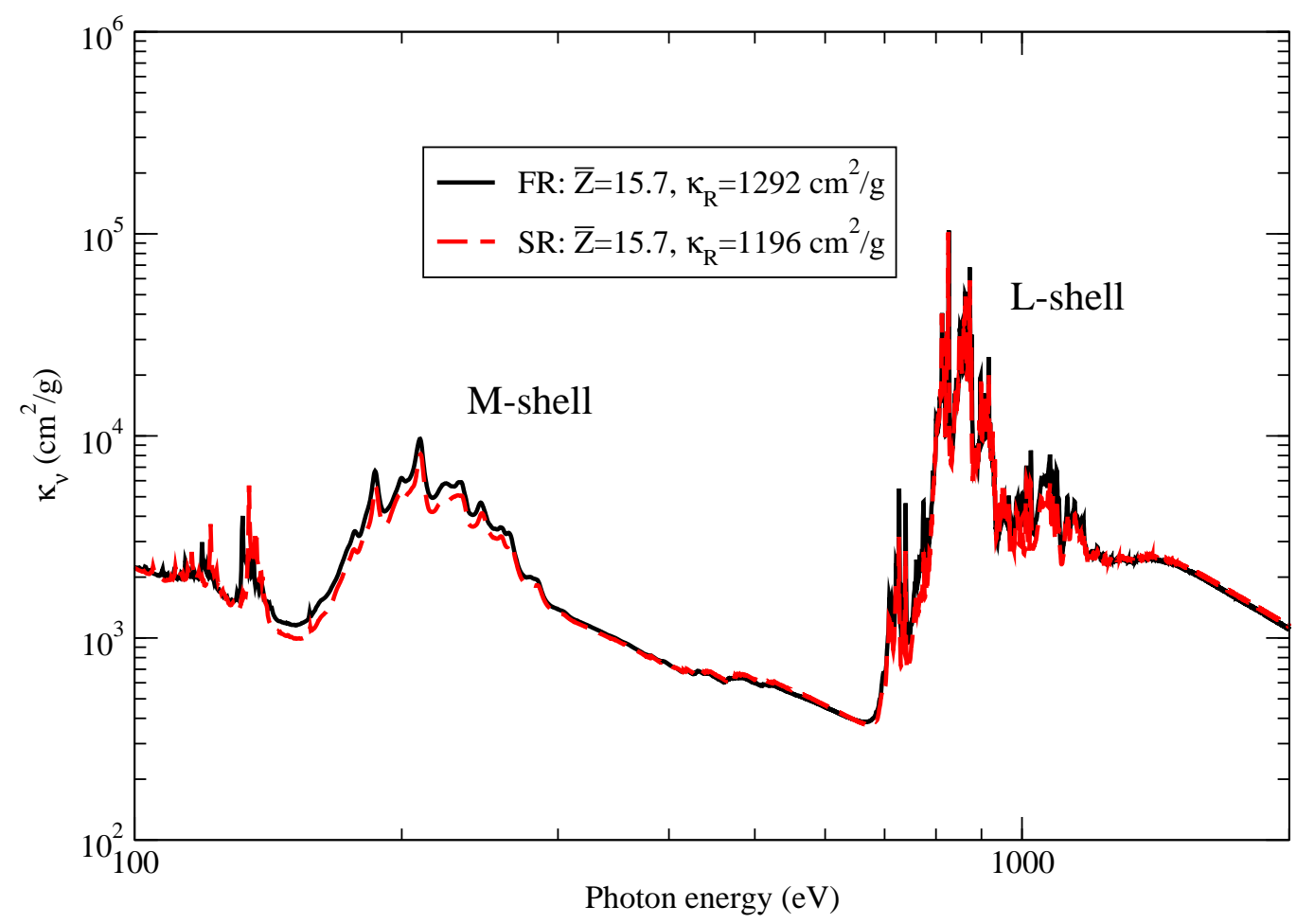

Figure 1: (Color online) Comparison of fully relativistic (FR; black solid line) and semirelativistic (SR; red dashed line) LTE monochromatic opacities of iron at $T=193 \mathrm{eV}$ and $N_{e}=10^{23}$ electrons $/ \mathrm{cm}^{3}$. The average charge states and Rosseland mean opacities are listed in the legend. The general locations of the L-shell and M-shell absorption features are also indicated.

when considering radiation transport at the base of the solar convection zone. The agreement between the two models for this quantity is not as perfect, showing a difference of $8 \%$. Since mean opacities depend on the details of the underlying monochromatic opacities, it is expected to see larger discrepancies in $\kappa_{\mathrm{R}}$ compared to $\bar{Z}$. For convenience, we reproduce the well-known formula for the Rosseland mean opacity,

$$
\frac{1}{\kappa_{\mathrm{R}}}=\frac{\int_{0}^{\infty} \kappa_{\nu}^{-1} \partial B_{\nu}(T) / \partial T d \nu}{\int_{0}^{\infty} \partial B_{\nu}(T) / \partial T d \nu},
$$

where $B_{\nu}(T)$ is the Planck function, $\nu$ is the photon frequency and $T$ is the temperature of the plasma. The difference in $\kappa_{\mathrm{R}}$ displayed in Fig. 1 can be understood by noting from Eq. (1) that the Rosseland mean is computed using 
an inverse, or harmonic, averaging scheme, and that the Rosseland weighting function, $\partial B_{\nu}(T) / \partial T$, peaks at a photon energy of approximately four times the temperature, which is $\sim 800 \mathrm{eV}$ in this case. This inverse weighting causes the lowest values of the monochromatic opacity in the vicinity of $800 \mathrm{eV}$ to most strongly contribute to the mean opacity. An inspection of Fig. 1 shows a minimum opacity to occur at $\sim 700 \mathrm{eV}$, where the Mshell bound-free absorption switches over to L-shell bound-bound absorption features. This minimum region of monochromatic opacity is quantitatively very similar in the two curves, resulting in the relatively small difference of $8 \%$ in the Rosseland mean opacities, despite the larger differences discussed above in other parts of the spectrum. In the next section, we provide a more in-depth analysis of a case for which the difference in mean opacity is larger.

\section{Nickel}

The LTE opacity of nickel, another iron-peak element, has been the focus of recent experimental and theoretical investigations due to its importance in the modeling of pulsations in stellar envelopes $[15,16]$. The temperatures of interest (15-40 eV) are significantly lower than those considered for the base of the solar convection zone described in the previous section, resulting in the presence of much lower charge states. These near-neutral ion stages contain more bound electrons and, consequently, are more challenging to calculate in an accurate manner from an atomic physics perspective.

In this section, we consider FR and SR calculations of nickel opacities at a temperature of $T=24 \mathrm{eV}$ and a mass density of $\rho=2 \times 10^{-3} \mathrm{~g} / \mathrm{cm}^{3}$, similar to the conditions considered in Ref. [15, 16]. The spectral features of interest are $\Delta n=0$ transitions with $n=3$ for a charge state distribution centered about the Ca-like stage. Thus a model was constructed for the Sthrough Cr-like stages, i.e. $\mathrm{Ni}^{4+}$ through $\mathrm{Ni}^{12+}$. Transitions with $\Delta n=0$ are typically more sensitive to configuration-interaction effects due to the presence of more levels that are close in energy, resulting in a larger degree of mixing among the basis states after diagonalization of the Hamiltonian has been performed.

As in the previous iron example, we again consider a model with $n_{\max }=4$. Due to the interest in $\Delta n=0$ transitions with $n=3$ for this case, the list of configurations was obtained by promoting up to six $n=3$ electrons within the ground configuration of each ion stage, allowing a maximum of one of those electrons to reside in the highest lying $n=4$ shell. This approach resulted in 
approximately 500000 fine-structure levels. In Fig. 2, we present a comparison of FR and SR LTE monochromatic opacities of nickel for the above-stated conditions of $T=24 \mathrm{eV}$ and a mass density of $\rho=2 \times 10^{-3} \mathrm{~g} / \mathrm{cm}^{3}$. Overall,

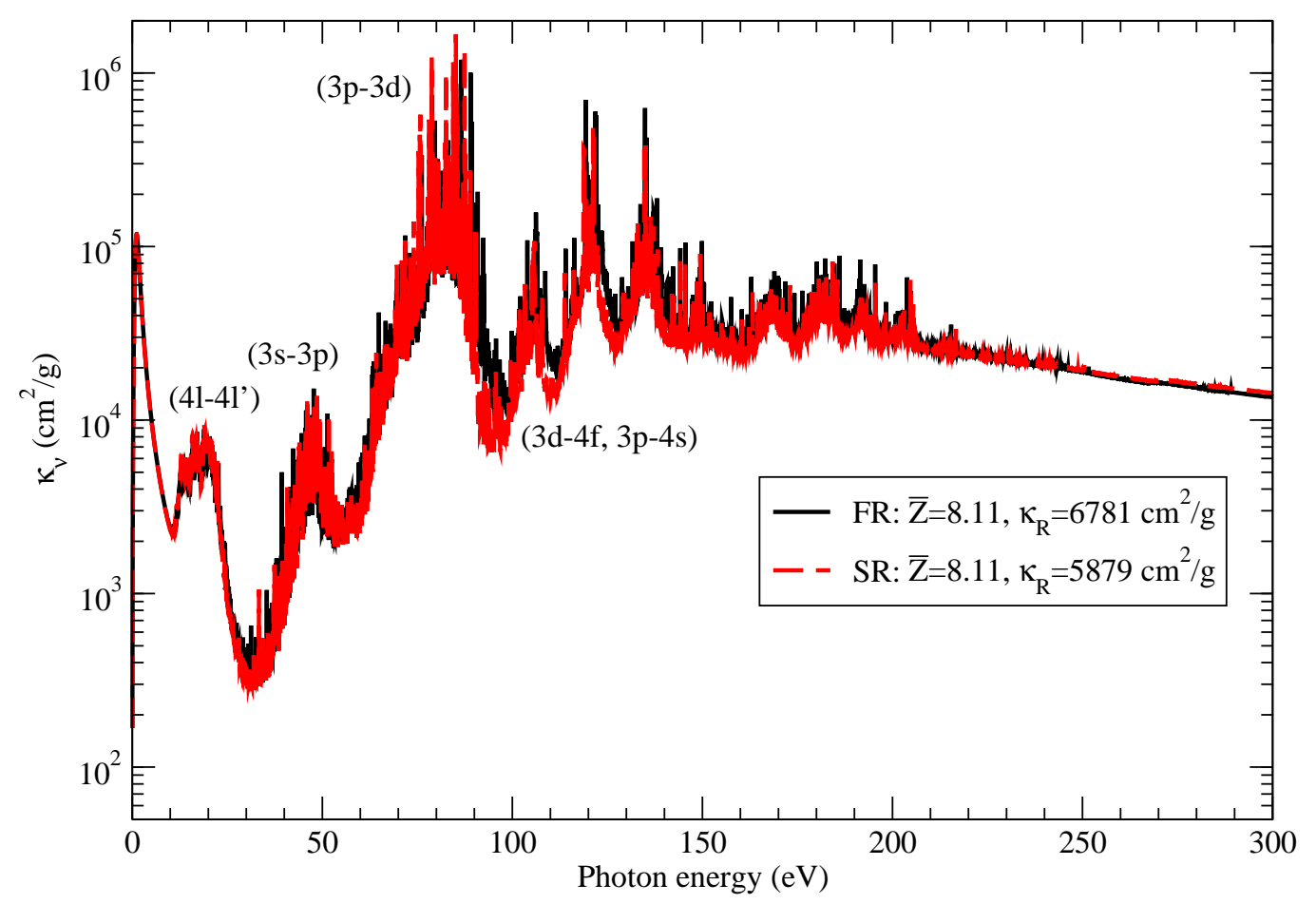

Figure 2: (Color online) Comparison of fully relativistic (FR; black solid line) and semirelativistic (SR; red dashed line) LTE monochromatic opacities of nickel at $T=24 \mathrm{eV}$ and $\rho=2 \times 10^{-3} \mathrm{~g} / \mathrm{cm}^{3}$. The average charge states and Rosseland mean opacities are listed in the legend. Orbital labels for the dominant transitions occurring in various features are also provided.

the agreement is good between the two curves, but the SR calculation is visibly lower in the troughs occurring at photon energies of 100 and $110 \mathrm{eV}$. The Rosseland mean opacity is once again an important quantity for this application and these local minima contribute strongly due to the harmonic weighting and the fact that the weighting function peaks at approximately $96 \mathrm{eV}$. Such differences in the monochromatic opacity result in an SR Rosseland mean opacity that is $15 \%$ smaller than the FR value.

In order to gain insight into the cause of these differences, a detailed study was performed to make the FR and SR calculations as similar as possible. Three items were identified in this regard. For the FR calculations, the small 
component of the bound and continuum Dirac wavefunctions represents an effect that is not captured in the SR approach. It is possible to remove the small component in the FR approach, resulting in what is referred to as a quasi-relativistic (QR) calculation $[2,17]$. For this particular example, changing from an FR to a QR calculation reduced the Rosseland mean opacity by $4 \%$, moving the results into closer agreement. For the SR calculation, the Hartree-Fock (HF) approach was changed to the more approximate HartreeFock-Slater (HFS) approach [12], which employs the (Slater) local-exchange approximation. This choice is consistent with the Dirac-Fock-Slater (DFS) approximation employed in the FR calculation $[2,17]$. Using the HFS approximation, instead of the HF approach, in the SR calculation increased the Rosseland mean opacity by $2 \%$, again bringing the FR and SR calculations into closer agreement.

The final modification to the SR calculations was to reset the semiempirical scale factors to one. As discussed by Cowan [12], these scale factors can be used to modify the radial integrals when constructing the Hamiltonian before the configuration-interaction diagonalization occurs. In this way, electron correlation that is not taken into account by the Hartree-Fock formalism can be included, at least in an approximate way, resulting in improved agreement with experimental energies. These scale factors are not included in the FR DFS calculations. Setting the scale factors to one in the HF calculations produced a $9 \%$ increase in the Rosseland mean opacity, moving the SR results even more in the direction of the $\mathrm{FR}$ and $\mathrm{QR}$ values.

A summary of the percentage changes in the Rosseland mean opacity due to the above modifications is presented in Table 1.

Table 1: A list of percentage changes in the Rosseland mean opacity of nickel at $T=24 \mathrm{eV}$ and $\rho=2 \times 10^{-3} \mathrm{~g} / \mathrm{cm}^{3}$ when comparing various calculations. The mean opacity of the "new" calculation is compared with that of the corresponding "baseline" calculation and the increase or decrease is presented in the final column as a percentage.

\begin{tabular}{ccc}
\hline $\begin{array}{c}\text { Baseline } \\
\text { calculation }\end{array}$ & $\begin{array}{c}\text { New } \\
\text { calculation }\end{array}$ & $\begin{array}{c}\text { Percentage change } \\
\text { (new vs. baseline) }\end{array}$ \\
\hline FR & QR & $-4 \%$ \\
SR (HF) & SR (HFS) & $+2 \%$ \\
SR (HF) & SR (HF, scale factors set to 1) & $+9 \%$ \\
\hline
\end{tabular}

In addition, we present in Fig. 3 a comparison of the $\mathrm{QR}$ and modified (HFS, with scale factors set to one) SR LTE monochromatic opacities of 
nickel for the same conditions as in Fig. 2. An inspection of the curves in

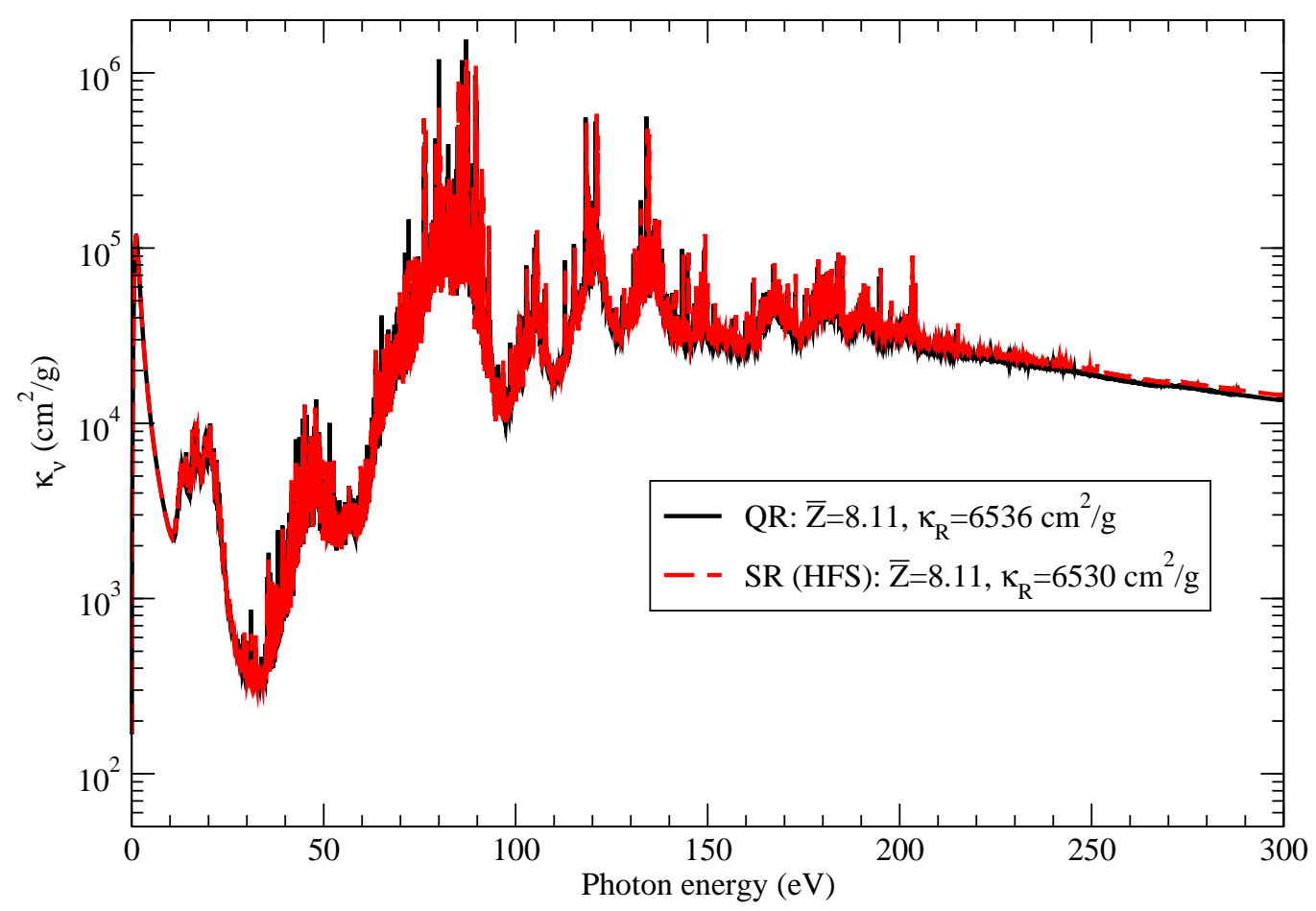

Figure 3: (Color online) Comparison of quasi-relativistic (QR; black solid line) and semirelativistic, HFS (SR (HFS); red dashed line) LTE monochromatic opacities of nickel at $T=24 \mathrm{eV}$ and $\rho=2 \times 10^{-3} \mathrm{~g} / \mathrm{cm}^{3}$. The SR (HFS) results were calculated with the scale factors set to one. See text for details. The average charge states and Rosseland mean opacities are listed in the legend.

Fig. 3 shows that much of the discrepancy in the local minima has been eliminated by making the two types of calculations as similar as possible, according to the above discussion. In addition, as indicated in the legend, the difference in Rosseland mean opacities has been reduced from $15 \%$ to $1 \%$. So, the source of the discrepancies between the default FR and SR calculations has been largely identified in this case. Additional calculations (not shown) indicate that increasing the size of the model to $n_{\max }=5$ increases the Rosseland mean opacity by approximately a factor of two, and a difference of 15-20\% persists between the FR and SR values when comparing the baseline models. In order to obtain a fully converged mean opacity, still higher values of $n_{\max }$ are required. Further study is necessary to understand what effect these differing opacities might have on the modeling of stellar envelopes. 


\section{Samarium}

While the previous two applications concerned the modeling of stars, in this section we consider the more exotic case of neutron star mergers (NSMs). NSMs are promising candidates for the observation of gravitational waves $[18,19]$. However, such mergers are also predicted to emit electromagnetic radiation [20,21,22], providing an alternative, independent method for detecting these events. The question of whether this electromagnetic radiation can be observed in the optical or infrared bands is a topic of current research. In order to model the light curves and spectra produced by NSMs, it is necessary to calculate the contribution to the radiative opacity from heavy elements, such as the lanthanides, that are expected to be present due to the capture of rapid neutrons during the $r$-process [23, 24]. This is a daunting task when attempting to carry out accurate atomic structure calculations due to the large number of bound electrons (e.g. $\sim 60-70$ for lanthanide elements) that exist for near-neutral ions, which can lead to a very complicated frequency-dependent opacity. For example, for ion stages with a half-filled $f$ shell in the ground configuration, the number of relevant bound-bound line features can exceed $10^{8}$ for the conditions of interest.

While it is impossible to calculate all such lines in spectroscopically accurate detail, the large gradients that are expected to exist in the material velocities of the ejecta region of a NSM provide some mitigation. Due to the motional Doppler shift associated with this rapidly expanding material, the line features will appear to be significantly broadened [23] to escaping photons, resulting in a quasi-continuum of opacity, rather than a discrete set of well-defined features. This smearing effect suggests that the line features need only to be calculated with approximate energy positions and strengths in order to obtain some insight into the type of electromagnetic signal that would be produced by a NSM.

Thus, in this section, we present a preliminary investigation of the LTE opacity of samarium ( $\mathrm{Sm}, Z=62$ ) obtained with the LASER codes at conditions relevant to NSM modeling. We consider temperatures in the vicinity of $T \sim 1 \mathrm{eV}$ and a mass density of $\rho=10^{-13} \mathrm{~g} / \mathrm{cm}^{3}$, similar to the conditions considered previously by Kasen et al. [23]. These conditions require an atomic physics model that contains the first four or so ion stages of Sm.

In order to model such ion stages with the LASER codes, it was necessary to implement logic in the RATS atomic structure code to take into account the seniority quantum number, $\nu$, when performing the angular momentum 
coupling to obtain the $J$-resolved basis states that are used to construct the Hamiltonian before diagonalization occurs. The seniority quantum number is required when four electrons occur in an $f$ or $g$ subshell of a particular configuration. (See, for example, Table 1 of Ref. [25].) As a specific example, consider the ground configuration of $\mathrm{Sm}^{2+}$, which can be written in the compact notation [Xe] $4 f^{4} 6 s^{2}$ using $n l$ orbital notation or [Xe] $4 f_{5 / 2}^{4} 6 s_{1 / 2}^{2}$ using $n l j$ orbital notation. Here, "[Xe]" indicates the completely full set of subshells associated with the xenon ground configuration. In generating excited-state configurations for this ion stage, a four-electron promotion produces the $[\mathrm{Xe}] 4 f_{7 / 2}^{4} 6 s_{1 / 2}^{2}$ configuration. Following the standard approach for the $j j$-coupling scheme, all possible $J$-resolved basis states that can arise from this configuration are obtained by first coupling the electrons within each $n l j$ subshell to obtain intermediate $J_{\text {int }}$ values. Then these intermediate values are coupled to obtain a final $J$ value for each basis state. For the $4 f_{7 / 2}^{4}$ subshell, there are seven possible intermediate $J_{\text {int }}$ values: $0,2,2,4,4,5$, 8. The duplicate values of $J_{\text {int }}=2$ and 4 are further distinguished by the seniority quantum number, $\nu$, which also takes on the integer values of 2 and 4 for this case. Thus, the intermediate $J_{\text {int }}$ values for the $4 f_{7 / 2}^{4}$ subshell can be denoted in the following unambiguous manner: $0,2(\nu=2), 2(\nu=4), 4$ $(\nu=2), 4(\nu=4), 5,8$. With such an appropriately defined set of $J$-resolved basis states, the energy degeneracy associated with configurations that contain a $4 f_{7 / 2}^{4}$ subshell is removed and the diagonalization of the Hamiltonian proceeds in the usual way to obtain a set of fine-structure levels and energies that include full configuration interaction.

Returning to the discussion of the Sm opacity, a relativistic model was generated for the first four ion stages of this element. Due to the relatively cold $(\sim 1 \mathrm{eV})$ conditions of interest, it was only necessary to include a relatively small number of low-lying configurations in each ion stage in order to obtain a converged set of fine-structure level populations as dictated by LTE Saha-Boltzmann statistics. This small number of configurations was used as input to the RATS atomic structure code, which generated on the order of $10^{5}$ levels and $3.3 \times 10^{8}$ lines, as indicated in Table 2 . In Table 3 , the RATS ionization energies are compared with those in the NIST database [26]. Discrepancies on the order of $15 \%$ are observed, indicating a possible method for improving the calculated Saha charge state distribution, which depends in a fundamental way on the ionization energies via the usual Boltzmann factor. The GIPPER code was used to generate the photoionization cross 
Table 2: A list of configurations, number of fine-structure levels, and number of absorption lines in the samarium model considered in this work.

\begin{tabular}{cccc}
\hline Ion stage & configurations & \# of levels & \# of lines \\
\hline $\mathrm{Sm}^{0+}$ & $4 f^{6} 6 s^{2}, 4 f^{5} 5 d^{1} 6 s^{2}, 4 f^{6} 5 d^{1} 6 s^{1}, 4 f^{6} 5 d^{2}$, & 60806 & 249301825 \\
& $4 f^{5} 5 d^{1} 6 s^{1} 6 p^{1}, 4 f^{6} 5 d^{1} 6 p^{1}, 4 f^{6} 6 s^{1} 6 p^{1}$ & & \\
$\mathrm{Sm}^{1+}$ & $4 f^{6} 6 s^{1}, 4 f^{6} 5 d^{1}, 4 f^{6} 6 p^{1}, 4 f^{5} 5 d^{2}$, & 29970 & 67743385 \\
& $4 f^{5} 5 d^{1} 6 s^{1}, 4 f^{5} 5 d^{1} 6 p^{1}, 4 f^{5} 6 s^{1} 6 p^{1}$ & & \\
$\mathrm{Sm}^{2+}$ & $4 f^{6}, 4 f^{5} 6 s^{1}, 4 f^{5} 5 d^{1}, 4 f^{5} 6 p^{1}$, & 13170 & 13318114 \\
& $4 f^{4} 5 d^{2}, 4 f^{4} 5 d^{1} 6 s^{1}, 4 f^{3} 5 d^{2} 6 s^{1}$ & & \\
$\mathrm{Sm}^{3+}$ & $4 f^{5}, 4 f^{4} 6 s^{1}, 4 f^{4} 5 d^{1}, 4 f^{4} 6 p^{1}$ & 1994 & 320633 \\
\hline
\end{tabular}

Table 3: Comparison of ionization energies for the first three ion stages of samarium. Values are presented for the FR approach described in this work and from the NIST database [26].

\begin{tabular}{ccc}
\hline Ion stage & \multicolumn{2}{c}{ Ionization energy $(\mathrm{eV})$} \\
\cline { 2 - 3 } & FR & NIST \\
\hline $\mathrm{Sm}^{0+}$ & 4.85 & 5.64 \\
$\mathrm{Sm}^{1+}$ & 10.6 & 11.1 \\
$\mathrm{Sm}^{2+}$ & 20.4 & 23.4 \\
\hline
\end{tabular}

sections that were used to obtain the bound-free contribution to the opacity. The ATOMIC code was then used to process these fundamental data to calculate the level populations and a monochromatic opacity. The scattering and free-free contributions to the opacity were obtained from the simple, analytic formulas [27] associated with Thomson and Kramers, respectively.

In Figure 4, we present the LTE opacity of Sm at $T=0.5 \mathrm{eV}$ and $\rho=10^{-13} \mathrm{~g} / \mathrm{cm}^{3}$. In this case, the lines were calculated with a Voigt profile, using the natural and thermal Doppler widths. (We note that the latter broadening is not the same as the previously discussed broadening associated with motional Doppler broadening, which is addressed below.) For comparison, the opacity associated with only free electrons is also provided in a separate panel. The inclusion of the line features dramatically increases the opacity in the optical range $(1.65-3.26 \mathrm{eV})$ by up to seven orders of magnitude. The absorption in the near-infrared range below $1.65 \mathrm{eV}(0.496-1.65 \mathrm{eV})$ is also greatly increased, by a few orders of magnitude in this case. These results suggest that light curves for NSMs would more likely be observed in 

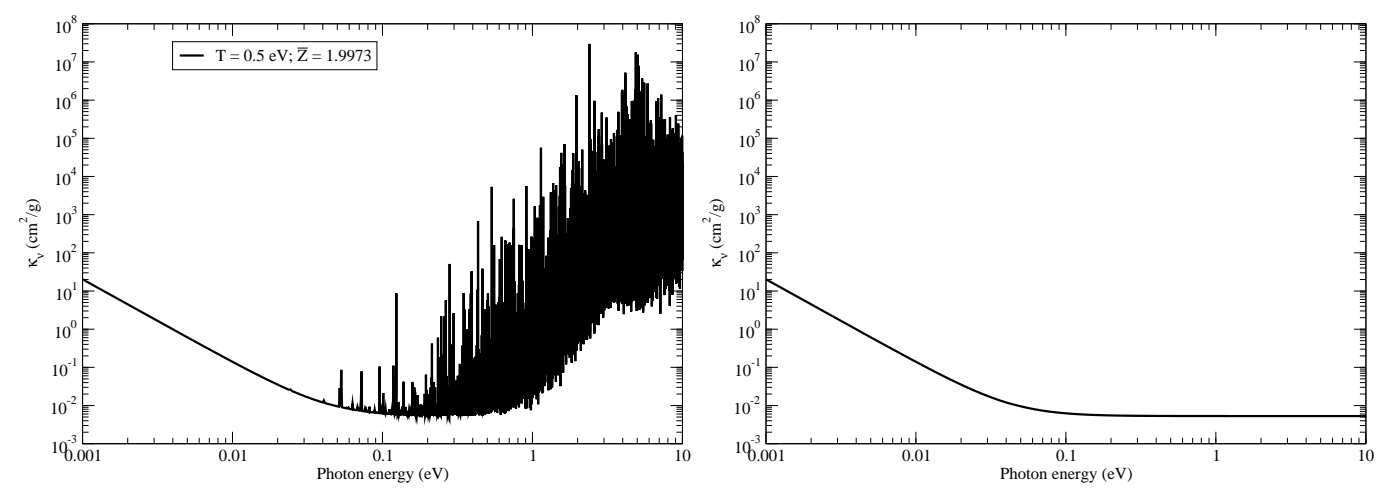

Figure 4: The LTE monochromatic opacity for samarium at $T=0.5 \mathrm{eV}$ and $\rho=$ $10^{-13} \mathrm{~g} / \mathrm{cm}^{3}$. The left panel displays the total opacity, while the right panel displays only the contributions due to free electrons, i.e the free-free and scattering contributions. The average charge state for these conditions is listed in the legend of the left panel.

the mid-infrared range $(0.124-0.496 \mathrm{eV})$ compared to the near-infrared and optical bands.

In order to simulate the effect of the motional Doppler shift described above, we recomputed the Sm opacity at the above conditions, but with an enhanced Dopper width that corresponds to an effective temperature of $T_{\text {eff }}=$ $20 \mathrm{MeV}$. This very high temperature was used only in the Doppler-width formula and corresponds to a velocity gradient of $\Delta v / c \sim \Delta \nu / \nu \sim 0.01$, where $c$ is the speed of light. The Sm opacity that results from this calculation is represented in Figure 5 as the dashed red curve, which is overlayed on the solid black curve from Figure 4. As expected, the Doppler-enhanced curve is somewhat smoother than the one calculated in the standard manner. We also note that the area under the broadened curve is significantly higher than that under the standard curve for energies below about $1 \mathrm{eV}$. This difference indicates that the standard curve contains very narrow lines that are not resolved with the chosen grid spacing of photon energies, and so opacity is actually lost in that calculation due to this deficiency. In the case with enhanced broadening, the line widths are sufficiently broad that all lines are adequately resolved and we obtain a faithful representation of the monochromatic opacity within the enhanced-broadening approximation.

Next, we generated a table of these Doppler-enhanced Sm opacities on a temperature-density grid for use in generating a NSM light curve. While the ejecta region is expected to contain all of the lanthanide elements in varying abundances, Sm was used in this preliminary investigation as a surrogate to 


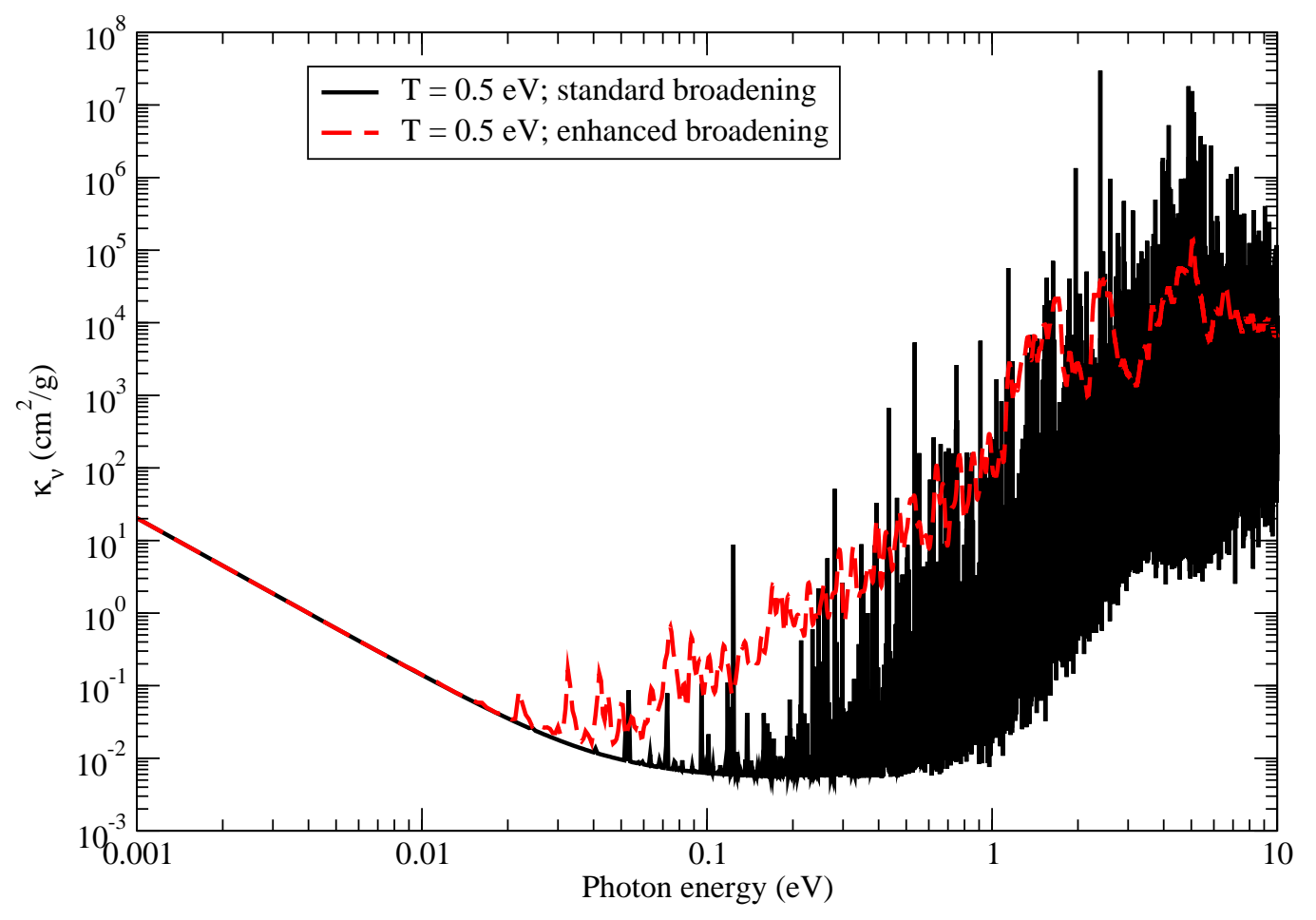

Figure 5: (Color online) The same as the left panel in Figure 4, except that an additional, dashed red curve is displayed. The dashed curve represents the calculation performed with enhanced broadening for the bound-bound features in order to simulate the effect of motional Doppler broadening. See text for details.

represent the entire lanthanide contribution to the opacity. The temperature grid consisted of 13 values ranging from $0.01-5.0 \mathrm{eV}$ : $0.01,0.07,0.1,0.5,1.0$, $1.5,2.0,2.5,3.0,3.5,4.0,4.5$ and $5.0 \mathrm{eV}$. The density grid consisted of 8 values ranging from $10^{-20}-10^{-13} \mathrm{~g} / \mathrm{cm}^{3}$ in steps of $10 \mathrm{~g} / \mathrm{cm}^{3}$.

To calculate the light curves (emission in a given band as a function of time), we employ the ejecta produced in the merger of two $1.4 \mathrm{M}_{\odot}$ neutron stars from the simulations of Ref. [28]. Assuming the ejected material is ballistic, we calculate the evolution of the ejecta position in 3-dimensions and density. The temperature is evolved including the PdV work done during the expansion, energy deposition from the radioactive decay of $r$-process elements using the approximation developed by Metzger et al. [29], and radiative cooling. The ejected material is roughly axisymmetric and, for our detailed post-process radiative transfer, we place this material on a 2-dimensional ax- 
isymmetric grid. The post-process transport is calculated for each timestep along line trajectories using the monochromatic opacities in the absorption approximation, producing full spectra along different lines-of-sight. We then bin these spectra into 3 separate wavelength bands given by the optical, near-infrared, and mid-infrared ranges defined above.

In Figure 6, we present light curves generated with the above method for the merger of two $1.4 \mathrm{M}_{\odot}$ neutron stars. The solid curves represent the edge-on emission and the dotted curves represent the face-on emission, with respect to the plane of rotation. The emission is separated into the optical, near-infrared and mid-infrared bands, defined previously. There are several noteworthy trends in this figure. For example, the similarity of the edge-on and face-on emission indicates that the light-curve emission is more isotropic, and hence more likely to be observed, than the accompanying gamma-ray bursts that are expected to be preferentially emitted along the axis of rotation. As for the different wavelength bands, the most likely type of radiation to be observed is dictated by the longest time duration rather than peak flux. Thus, based on the present modeling, the mid-infrared emission is predicted to dominate the optical and near-infrared contributions to the NSM light curve, in agreement with the earlier analysis of the bound-bound absorption features displayed in the monochromatic opacity of Sm in Figures 4 and 5. This preliminary conclusion is also in accord with that of Kasen et al. [23].

\section{Conclusions}

The Los Alamos suite of relativistic atomic physics codes has been used to generate LTE opacities for the modeling of a variety of astrophysical plasmas. Comparisons between these fully relativistic calculations and their semi-relativistic counterparts for iron-peak elements displayed reasonably good agreement. Discrepancies on the order of $15 \%$ between the two sets of calculations for nickel were found to be caused by relativistic effects, the local-exchange approximation, and empirical scale factors. Samarium opacities were used to represent the entire lanthanide contribution to the opacity in the simulation of NSM light curves. These preliminary results indicate that the light curves will be dominated by emission in the infrared, rather than optical, bands. 


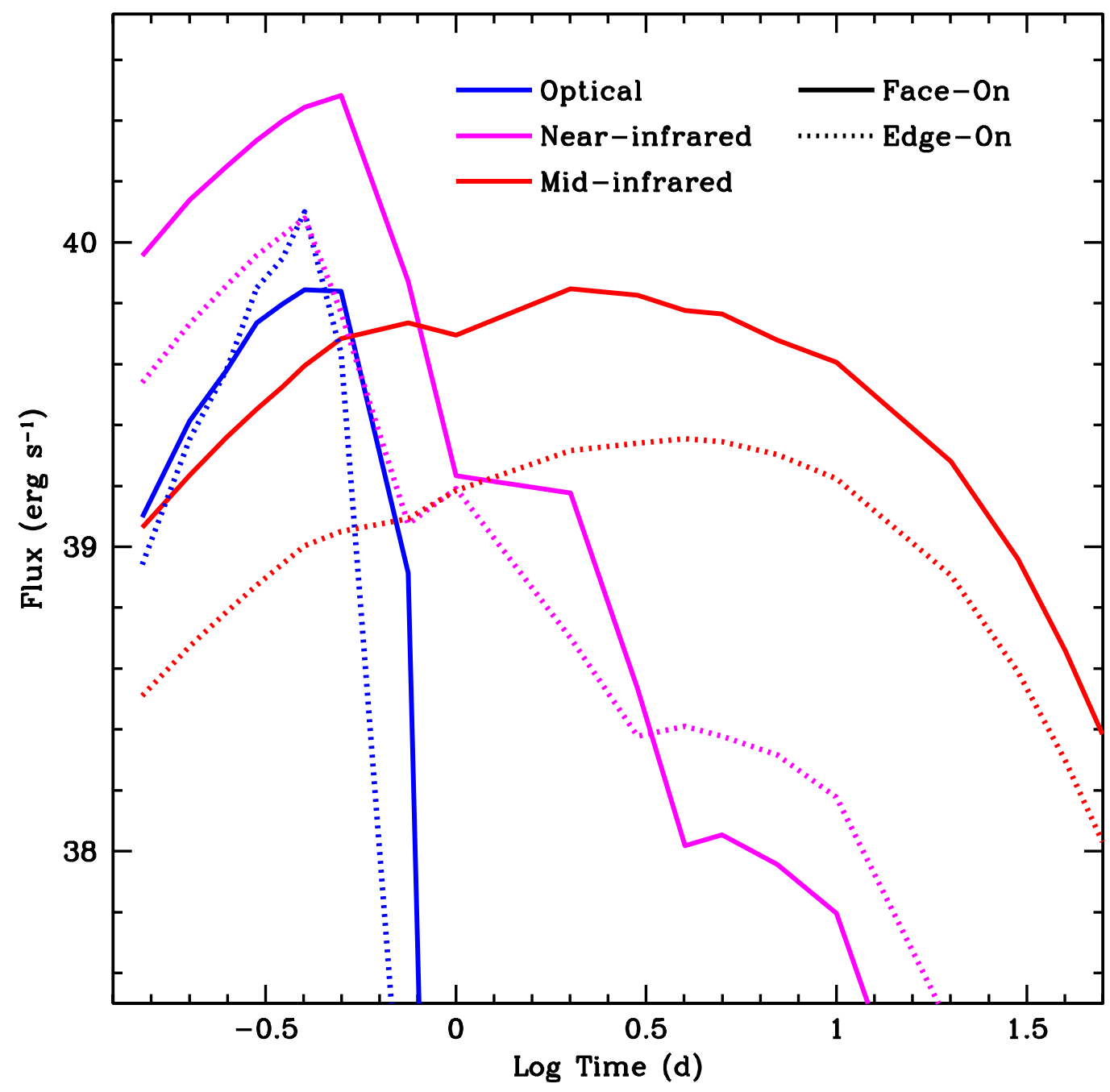

Figure 6: (Color online) Light curves for the merger of two 1.4 solar mass neutron stars. The emitted flux is presented for three bands of electromagnetic radiation: optical (1.65$3.26 \mathrm{eV}$; blue curves), near-infrared (0.496-1.65 eV; magenta curves) and mid-infrared (0.124-0.496 eV; red curves). Each set of three curves is further delineated according to whether the emission is observed face-on (solid curves) or edge-on (dotted curves) with respect to the plane of rotation. 


\section{Acknowledgments}

This work was performed under the auspices of the U.S. Department of Energy by Los Alamos National Laboratory under Contract No. DE-AC5206NA25396.

\section{References}

[1] C.J. Fontes, H.L. Zhang, J. Abdallah, Jr, R.E.H Clark, D.P. Kilcrease, J. Colgan, R.T. Cunningham, P. Hakel, N.H. Magee, M.E. Sherrill, accepted for publication in J. Phys. B (2015).

[2] D.H. Sampson, H.L. Zhang, C.J. Fontes, Phys. Rep. 477 (2009) 111.

[3] N.H. Magee, J. Abdallah, Jr, J. Colgan, P. Hakel, D.P. Kilcrease, S. Mazevet, M. Sherrill, C.J. Fontes, H.L. Zhang 2004 in 14 th Topical Conference on Atomic Processes in Plasmas edited by J S Cohen, S Mazevet and D P Kilcrease (AIP: New York) pp. 168-179.

[4] P. Hakel, D.P. Kilcrease 2004 in 14th Topical Conference on Atomic Processes in Plasmas edited by J S Cohen, S Mazevet and D P Kilcrease (AIP: New York) pp. 190-202

[5] P. Hakel, M.E. Sherrill, S. Mazevet, J. Abdallah, Jr, J. Colgan, D.P. Kilcrease, N.H. Magee, C.J. Fontes, H.L. Zhang, J. Quant. Spec. Rad. Trans. 99 (2006) 265.

[6] S. Bernitt, et al., Nature 492 (2012) 225

[7] G.V. Brown, Can. J. Phys. 86 (2008) 199.

[8] N.S. Oreshkina, S.M. Cavaletto, C.H. Keitel, Z. Harman, Phys. Rev. Lett. 113 (2014) 143001.

[9] S.D. Loch, C.P. Ballance, Y. Li, M. Fogle, C.J. Fontes, Astrophys. J. Lett. 801 (2015) L13.

[10] J.E. Bailey et al., Nature 517 (2015) 56.

[11] J. Abdallah, R.E.H. Clark, G. Csanak, R.D. Cowan, Los Alamos National Laboratory, Los Alamos Manual No. LA 11436-M-I (1988). 
[12] R.D. Cowan, Theory of Atomic Structure and Spectra, (U. Calif. Press, Berkeley, 1981).

[13] N.H. Magee, J. Abdallah, Jr., R.E.H. Clark, J.S. Cohen, L.A. Collins, G. Csanak, C.J. Fontes, A. Gauger, J.J. Keady, D.P. Kilcrease, A.L. Merts, Astron. Soc. Pac. Conf. Ser. 78 (1995) 51.

[14] J. Colgan, D.P. Kilcrease, N.H. Magee, Jr, G.S.J. Armstrong, J. Abdallah, Jr., M.E. Sherrill, C.J. Fontes, H.L. Zhang, P. Hakel, High Energy Density Phys. 9 (2013) 369.

[15] D. Gilles, et al., High Energy Density Phys. 7 (2011) 312.

[16] S. Turck-Chièze et al., High Energy Density Phys. 9 (2013) 473.

[17] D.H. Sampson, H.L. Zhang, A.K. Mohanty, R.E.H. Clark, Phys. Rev. A 40 (1989) 604.

[18] G.M. Harry, et al., Class. Quantum Gravity 27 (2010) 084006.

[19] F. Acernese, et al., Class. Quantum Gravity 23 (2006) S63.

[20] L.-X. Li, B. Paczyński, Astrophys. J. 507 (1998) L59.

[21] S. Rosswog, Astrophys. J. 634 (2005) 1202.

[22] B.D. Metzger, G. Martínez-Pinedo, S. Darbha, E. Quataert, A. Arcones, D. Kasen, R. Thomas, P. Nugent, I.V. Panov, N.T. Zinner, Mon. Not. R. Astron. Soc. 406 (2010) 2650.

[23] D. Kasen, N.R. Badnell, J. Barnes, Astrophys. J. 774 (2013) 25.

[24] J. Barnes, D. Kasen, Astrophys. J. 775 (2013) 18.

[25] I.P. Grant, B.J. McKenzie, P.H. Norrington, D.F. Mayers, N.C. Pyper, Comp. Phys. Comm. 21 (1980) 207.

[26] A. Kramida, Yu. Ralchenko, J. Reader, NIST ASD Team (2014), NIST Atomic Spectra Database (ver. 5.2), [Online]. Available: http://physics.nist.gov/asd [2014, September 1].

[27] W.F. Huebner, W.D. Barfield, Opacity, (Springer, New York, 2014). 
[28] O. Korobkin, S. Rosswog, A. Arcones, C. Winteler, Mon. Not. Roy. Astro. Soc. 426, 1940 (2102).

[29] B. D. Metzger, G. Martínez-Pinedo, S. Darbha, E. Quataert, A. Arcones, D. Kasen, R. Thomas, P. Nugent, I.V. Panov, N.T. Zinner, Mon. Not. Roy. Astro. Soc. 406, 2650 (2010). 